\title{
Breast carcinoma malignancy grading by Bloom-Richardson system vs proliferation index: reproducibility of grade and advantages of proliferation index
}

\author{
John S Meyer ${ }^{1}$, Consuelo Alvarez ${ }^{2}$, Clara Milikowski², Neal Olson ${ }^{3}$, Irma Russo ${ }^{4}$, \\ Jose Russo ${ }^{4}$, Andrew Glass ${ }^{3}$, Barbara A Zehnbauer ${ }^{5}$, Karen Lister ${ }^{6}$ and \\ Reza Parwaresch ${ }^{7}$ for the Cooperative Breast Cancer Tissue Resource
}

${ }^{1}$ St Luke's Hospital, Chesterfield, MO, USA; ${ }^{2}$ University of Miami, Miami, FL, USA; ${ }^{3}$ Kaiser Permanente, Northwest Region, Portland, OR, USA; ${ }^{4}$ Fox Chase Cancer Center, Philadelphia, PA, USA; ${ }^{5}$ Washington University, St Louis, MO, USA; ${ }^{6}$ Forest Park Hospital, St Louis, MO, USA; ${ }^{7}$ University of Kiel, Germany

\begin{abstract}
Questions of reproducibility and efficacy of histologic malignancy grading relative to alternative proliferation index measurements for outcome prediction remain unanswered. Microsections of specimens from the Cooperative Breast Cancer Tissue Resource (CBCTR) were evaluated by seven pathologists for reproducibility of grade and classification. Nuclear figure classification was assessed using photographs. Grade was assigned by the Bloom-Richardson method, Nottingham modification. Proliferation index was evaluated prospectively by deoxyribose nucleic acid precursor uptake with thymidine (autoradiographic) or bromodeoxyuridine (immunohistochemical) labeling index using fresh tissue from 631 node-negative breast cancer patients accessioned at St Luke's Hospital. A modified Nottingham-Bloom-Richardson grade was derived from histopathologic data. Median post-treatment observation was 6.4 years. Agreement on classification of nuclear figures $(N=43)$ was less than good by kappa statistic $(\kappa=0.38)$. Grade was moderately reproducible in four trials $(N=10,10,19,10)$ with CBCTR specimens $(\kappa=0.50-0.59)$. Of components of Bloom-Richardson grade, agreement was least for nuclear pleomorphism $(\kappa=0.37-0.50)$, best for tubularity $(\kappa=0.57-0.83)$, and intermediate for mitotic count $(\kappa=0.45-0.64)$. Bloom-Richardson grade was a univariate predictor of prognosis in node-negative St Luke's patients, and was improved when mitotic count was replaced by labeling index (low, mid, or high). When labeling index was added to a multivariate model containing tumor size and vessel invasion, grade was no longer a significant predictor of tumor-specific relapse-free or overall survival. Mitotic index predicted best when intervals were lowered to $0-2,3-10$, and $>10$ mitotic figures per ten $0.18 \mathrm{~mm}^{2}$ highpower fields. We conclude that Nottingham-Bloom-Richardson grades remain only modestly reproducible. Prognostically useful components of grade are mitotic index and tubularity. The Nottingham-BloomRichardson system can be improved by lowering cutoffs for mitotic index and by counting 20-30 rather than 10 high-power fields. Measurement of proliferation index by immunohistochemically detectable markers will probably give superior prognostic results in comparison to grade.

Modern Pathology (2005) 18, 1067-1078. doi:10.1038/modpathol.3800388; published online 13 May 2005
\end{abstract}

Keywords: Bloom-Richardson; breast neoplasms; bromodeoxyuridine; cell kinetics; grade; proliferation; reproducibility

Wide spectrums of morphologic patterns and clinical behavior in breast carcinomas have led to many attempts to create meaningful classifications. Green-

Correspondence: Dr JS Meyer, MD, Department of Pathology, St Luke's Hospital, 232 South Woods Mill Road, Chesterfield, MO 63017, USA.

E-mail: john.meyer@stlukes-stl.com

Received 19 November 2004; revised 5 January 2005; accepted 7 January 2005; published online 13 May 2005 ough $^{1}$ introduced breast carcinoma grading in 1925. He used seven histologic characteristics to classify breast carcinomas into three grades of malignancy. Patey and Scarff ${ }^{2}$ in 1928 selected tubule formation, variation in nuclear size, and nuclear hyperchromatism as principal variables that were modified by evidence of secretion (good) and mitotic frequency (bad). In 1957, Bloom and Richardson ${ }^{3}$ proposed a simplified system which utilized only three of Greenough's variables: gland-formation (tubularity), 
degree of variation in nuclear size and shape (pleomorphism), and 'hyperchromatic figures' as an estimate of proliferation. Despite lack of specific criteria for quantifying variables measured, the grading system was effective for prognosis. Modifications to enhance reproducibility of scores resulted in the Nottingham-Bloom-Richardson system, ${ }^{4,5}$ which has been endorsed by the College of American Pathologists and the World Health Organization. ${ }^{6}$ The basic principle has remained summation of scores for the three variables, each of which is assigned from one to three points according to degree of departure from normal breast epithelium. A total score of 5 or less defines grade 1, 6-7 points grade 2 , and 8-9 points grade 3 . Rationale for an unweighted combined scoring of these three correlated variables has never been put forth, but a relationship between grade of invasive ductal carcinoma (IDC) of the breast and outcome has been recognized consistently. ${ }^{3,4,7-9}$ Strength of the prognostic relationship has fallen short of levels necessary for critical therapeutic decisions, and reproducibility of grading has remained a matter of concern ${ }^{10-19}$ (Table 1). The Cooperative Breast Cancer Tissue Resource (CBCTR) has collected and categorized 9000 breast cancer specimens available as formalin-fixed blocks in paraffin. ${ }^{20}$ Histologic malignancy grading could facilitate exploitation of the resource by investigators. Thus, we set out to evaluate reproducibility of breast carcinoma grading of CBCTR specimens. Efficacy of grading vs proliferation index (PI) for prediction of outcome was evaluated in a second set of patients.

\section{Materials and methods}

\section{Populations Studied}

\section{Grading reproducibility}

We used specimens selected from a previously ungraded CBCTR set of 9000 breast carcinoma specimens from years 1982 to 1990 to assess reproducibility of grading. CBCTR specimens consist of paraffin-embedded, formalin-fixed tissue blocks and microsections stored in participating institutions where the patients were diagnosed and treated.

Assessment of relationships between grade, proliferation index, and outcome

A separate set of 631 node-negative infiltrating breast carcinomas was used to evaluate prognostic efficacy of grade and other variables. At least five axillary lymph nodes were examined microscopi-

Table 1 Breast carcinoma grading: levels of agreement reported in prior studies

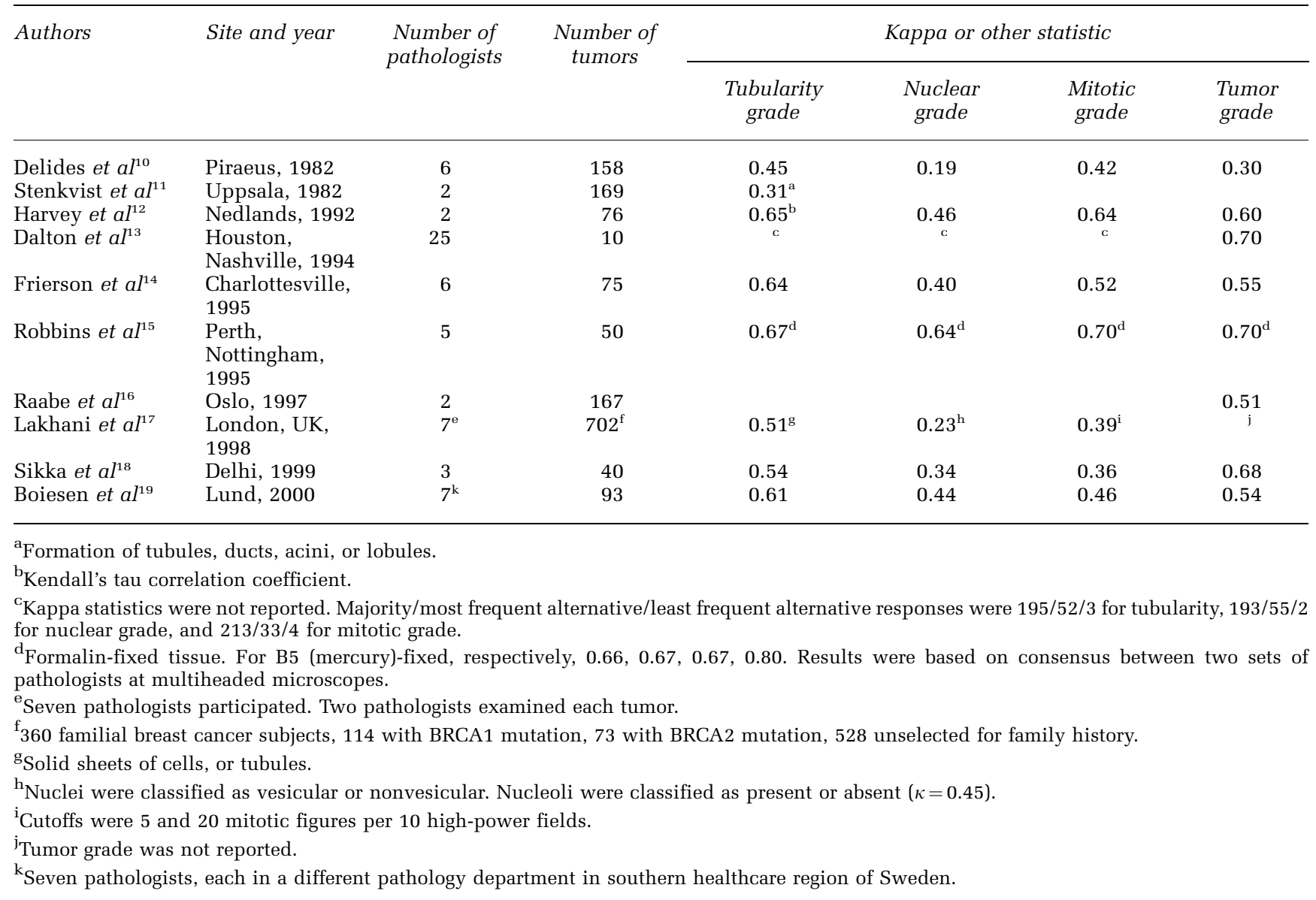


cally in each patient. The specimens were accessioned serially at St Luke's Hospital in the years 1985-1998. Patients with negative axillary lymph nodes were selected because assessment of prognosis may influence decisions to recommend surgical adjuvant therapy in this group.

These patients were selected consecutively from a larger set that had been evaluated prospectively for proliferation index by DNA precursor uptake by fresh tissue slices. All had unifocal invasive carcinomas of known size. Breast-conserving surgery followed by radiation to the residual breast was performed in $56.6 \%$, and total mastectomy in $43.4 \%$ of patients. The number of axillary nodes examined varied from 5 to 37 , mean 12.8 , median 12 . Therapy was individualized according to characteristics of the patient and tumor, particularly tumor size, grade, hormonal receptor assay results, and labeling index which, along with other variables, were available at time of initial treatment. High labeling index was associated with increased probability of adjuvant chemotherapy, and expression of estrogen and progesterone receptors with administration of tamoxifen.

The median period of observation after initial therapy was 6.4 years.

\section{Mitotic Figure Recognition}

Nuclear figures from 10 microsections used for Nottingham-Bloom-Richardson grading were photographed through a $\times 40$ dry objective lens with negative color film. Final magnification of prints was 500 diameters. A total of 50 nuclear figures were included and encircled with a wax pencil in the 36 prints provided to each participating pathologist. The participant was asked to classify each object as a mitotic figure (yes/no). If a mitotic figure, then phase of mitosis, and if not a mitotic figure, then resting cell, apoptosis, pyknosis, necrosis, or other was to be specified. The identities assigned by the photographer are listed in Table 2.

\section{Tumor Grading}

\section{Cooperative Breast Cancer Tissue Resource} specimens

All grading reproducibility exercises were carried out using a separate set of glass slides representing

Table 2 Identities of objects photographed

\begin{tabular}{lr}
\hline Object type $^{\mathrm{a}}$ & Number \\
\hline Mitosis, prophase & 1 \\
Indefinite for prophase & 3 \\
Mitosis, nonprophase & 19 \\
Indefinite for mitosis, nonprophase & 2 \\
Apoptosis & 12 \\
Pyknosis or hyperchromatic nucleus & 6
\end{tabular}

${ }^{\mathrm{a}}$ As identified by photographer. new invasive carcinomas for each exercise. Seven pathologists participated, five of them in all five trials. Mean age of pathologists is 58.6 years, median 62, range 47-74. Their mean number of years in practice is 25.7 years, median 24, range 13-44. Six pathologists had special interests in breast pathology. They graded the tumors individually without intercommunication according to the Nottingham-Bloom-Richardson system. ${ }^{5}$ Cellular, well-fixed fields from peripheral tumor regions were utilized. The first of 10 consecutive highpower fields in which mitotic figures were counted was selected without constraint that a mitotic figure was or was not present. Five trials were run in which a single microsection from each breast carcinoma was evaluated by each participant for tumor grade. No special instructions were given prior to trial 1. After the first trial, the group met to discuss sources of disagreement, review the Nottingham-Bloom-Richardson system, and assure that mitotic counts were adjusted according to standard microscope field area. After the second trial, another meeting was held to discuss discrepancies. Six of the original seven pathologists participated in two more trials using invasive carcinomas. Before the third trial, pathologists reviewed criteria for mitotic figures and recognition of prophase mitosis using a microscope for multiple viewers.

\section{St Luke's Hospital specimens}

Tumors were graded prospectively by the pathologist responsible for diagnosis. In all, 10 pathologists were active during the period the specimens were accessioned. Tubularity was scored as 1 if $<5 \%$ of tumor cells participated in formation of lumens, 2 if $5-50 \%$ participated, and 3 if $>50 \%$ participated. Nuclear grade was assigned as 1 if nuclei were predominantly less than $10 \mu \mathrm{m}$ in greatest diameter, 3 if predominantly $>13 \mu \mathrm{m}$ in diameter, and 2 if intermediate. Calibrated ocular grids were used as size references. Mitosis was defined as prophase (uniformly condensed chromosomes), metaphase, anaphase, or telophase. Hyperchromatic nuclei and apoptotic figures were excluded. Mitotic indices were recorded prospectively per $10 \times 40$ highpower fields, field area approximately $0.18 \mathrm{~mm}^{2}$, and recorded in four categories: $0-2,3-5,6-10$, and $>10$. These cutoffs were used to accommodate the known lognormal distribution of proliferation index, ${ }^{21}$ but do not correspond exactly to NottinghamBloom-Richardson cutoffs. ${ }^{5}$ The exact numerical score per 10 high-power fields was also recorded for 404 tumors. Grades were computed from the individual scores using the cutoffs stated above for tubularity and nuclear size. Intervals of $0-2,3-10$, and $>10$ were used for mitotic index because they most closely divided the population into three groups of equal size. 


\section{Assessment of Proliferation Index by DNA Precursor Uptake}

Proliferation index was measured as DNA labeling index by in vitro tritiated thymidine (1985-1987), or 5-bromo-2'-deoxyuridine (bromodeoxyuridine) uptake (1987-1998) as previously described..$^{22}$ Proliferation index was measured on approximately $95 \%$ of primary breast carcinoma specimens accessioned during the time of the study. Omissions occurred because of very small tumors or on rare occasion technical error. One of the 631 carcinomas with recorded proliferation index was excluded from the analysis because only in situ carcinoma was available in the specimen on which the assay was performed.

\section{Statistical Analysis and Ethical Review}

Levels of agreement among pathologists were assessed by concordance proportion $(C=$ number of cases on which two specified pathologists agreed/ number of cases) and by the kappa $(\kappa)$ statistic wherein $<0$ is interpreted as poor, $0-0.2$ as slight, 0.2-0.4 fair, 0.4-0.6 moderate, 0.6-0.8 good, and $>0.8$ almost perfect. ${ }^{23}$

Survival intervals were calculated from date of definitive surgery. Relapse was defined as clinically evident recurrent tumor in any site other than in ipsilateral or contralateral breast. Survival analysis was performed by the Kaplan-Meier product limit method $^{24}$ with evaluation by log-rank test. Patients without relapse were censored at time of last information if alive or at death if tumor-free. Variables with univariate prognostic significance at $P<0.05$ in two-tailed tests and patient's age were compared by Cox's proportional hazards multivariate analysis using the statistical package JMP version 5.0 (SAS Institute, Inc., Cary, NC, USA).

The study was approved by the Review Board for Human Subjects Research of St Luke's Hospital.

\section{Results}

\section{Nuclear Figure Classification}

Seven pathologists examined 43 nuclear figure photographs. Yes/no scoring for mitosis showed a fair level of agreement with $C_{\text {avg }}=0.69, \kappa_{\text {avg }}=0.38$. Agreement for type of mitotic figure was moderate $\left(C_{\text {avg }}=0.73, \kappa_{\text {avg }}=0.54\right)$, while agreement for type of nonmitotic hyperchromatic figure was also only fair $\quad\left(C_{\mathrm{avg}}=0.38, \quad \kappa_{\mathrm{avg}}=0.23\right)$. Unanimous agreement was reached for 14 objects of which four were classified as mitotic figures and 10 as not. Six of seven pathologists agreed on another 10 objects of which two were nearly unanimous for and eight against mitosis. Concordance proportions for pairs of pathologists varied from 0.54 to 0.90. On scanning the individual responses, agreement appeared to be good for metaphase and anaphase. Two pathologists never designated a prophase. One pathologist recognized four of four prophases as classified under the microscope by the photographer, but only two others recognized any of these photographs as prophases. An en face metaphase, as classified by direct microscopy, was designated prophase by one pathologist. One telophase, as recognized by the photographer, was shown. Two pathologists designated it as telophase and four designated not a mitotic figure.

\section{Tumor Grading Reproducibility}

Results were similar in agreement among participants for the five separate trials (Table 3). Kappa statistics indicate moderate agreement on tubularity, fair to moderate agreement on nuclear pleomorphism, moderate agreement on mitotic grade, and moderate agreement on overall tumor grade. Although unanimous agreement was achieved less often than not, differences in scoring of more than one point were infrequent (Table 4). In the fourth and fifth trials, proportions of prophases were recorded. They varied from 0.1 to $3.4 \%$, mean $1.4 \%$ in trial 4 . Average pairwise concordance on proportion of mitotic figures that were in prophase was 0.37 , and average pairwise $\kappa$ was 0.13 (slight agreement). In the fifth trial, proportions of prophases varied from 0.2 to $3.2 \%$, mean $0.9 \%$. Average pairwise concordance was 0.41 , average pairwise $\kappa$ was 0.09 (slight agreement).

Table 3 Results of tumor grading by five to seven pathologists

\begin{tabular}{|c|c|c|c|c|c|}
\hline \multirow[t]{2}{*}{ Trial } & \multirow[t]{2}{*}{$\mathrm{N}$ cases/pathologists } & \multicolumn{4}{|c|}{ Concordance (C) and kappa (к) statistics for average pairwise comparisons ${ }^{\mathrm{a}}$} \\
\hline & & Tubularity & Nuclear pleomorphism & Mitotic grade & Tumor grade \\
\hline 1 & $10 / 6$ & $C=0.91, \kappa=0.83$ & $C=0.61, \kappa=0.37$ & $C=0.74, \kappa=0.54$ & $C=0.72, \kappa=0.59$ \\
\hline 2 & $10 / 7$ & $C=0.84, \kappa=0.73$ & $C=0.58, \kappa=0.27$ & $C=0.71, \kappa=0.45$ & $C=0.72, \kappa=0.58$ \\
\hline 3 & $19 / 6$ & $C=0.82, \kappa=0.57$ & $C=0.68, \kappa=0.50$ & $C=0.68, \kappa=0.46$ & $C=0.67, \kappa=0.50$ \\
\hline 4 & $10 / 5$ & $C=0.84, \kappa=0.74$ & $C=0.55, \kappa=0.32$ & $C=0.84, \kappa=0.64$ & $C=0.74, \kappa=0.57$ \\
\hline 5 & $23 / 7$ & $C=0.85, \kappa=0.64$ & $C=0.65, \kappa=0.46$ & $C=0.83, \kappa=0.67$ & $C=0.70, \kappa=0.55$ \\
\hline
\end{tabular}

\footnotetext{
${ }^{\mathrm{a}}$ Kappa scores denote levels of agreement: $0-0.2=$ slight, $0.21-0.4=$ fair, $0.41-0.6=$ moderate, $0.61-0.8=$ substantial, $0.81-1=$ almost perfect
} 


\section{Outcome Assessment}

Cause-specific relapse-free survival analyses are shown in Figures 1-3. Cutoffs for covariates in survival plots are listed in Table 5. Univariate

Table 4 Detailed breakdown of agreement among seven pathologists, trial 2

\begin{tabular}{|c|c|c|c|c|c|}
\hline Characteristic & $\begin{array}{l}\text { Mean } \\
\text { score }\end{array}$ & Range & $\begin{array}{l}\text { Maximum } \\
\text { difference }^{\mathrm{a}}\end{array}$ & $\begin{array}{c}\text { Mean } \\
\text { concordance }\end{array}$ & $\begin{array}{c}\text { Kappa } \\
\text { statistic }\end{array}$ \\
\hline Tubularity & 2.3 & $1-3$ & $1^{\mathrm{b}}$ & 0.84 & 0.73 \\
\hline $\begin{array}{l}\text { Nuclear } \\
\text { pleomorphism }\end{array}$ & 2.5 & $1-3$ & $2^{\mathrm{c}}$ & 0.58 & 0.27 \\
\hline Mitotic index & 1.6 & $1-3$ & $2^{\mathrm{d}}$ & 0.71 & 0.45 \\
\hline Grade & 2.0 & $1-3$ & $1^{\mathrm{e}}$ & 0.72 & 0.58 \\
\hline
\end{tabular}

${ }^{\mathrm{a}}$ Maximum difference in grade.

${ }^{\mathrm{b}}$ Occurred 3 times; unanimous agreement occurred 7 times.

${ }^{\mathrm{c}}$ Occurred once; unanimous agreement occurred once.

${ }^{\mathrm{d}}$ Occurred 4 times; unanimous agreement occurred 5 times (all lowest mitotic grade).

${ }^{\mathrm{e}}$ Occurred 7 times; unanimous agreement occurred 3 times.
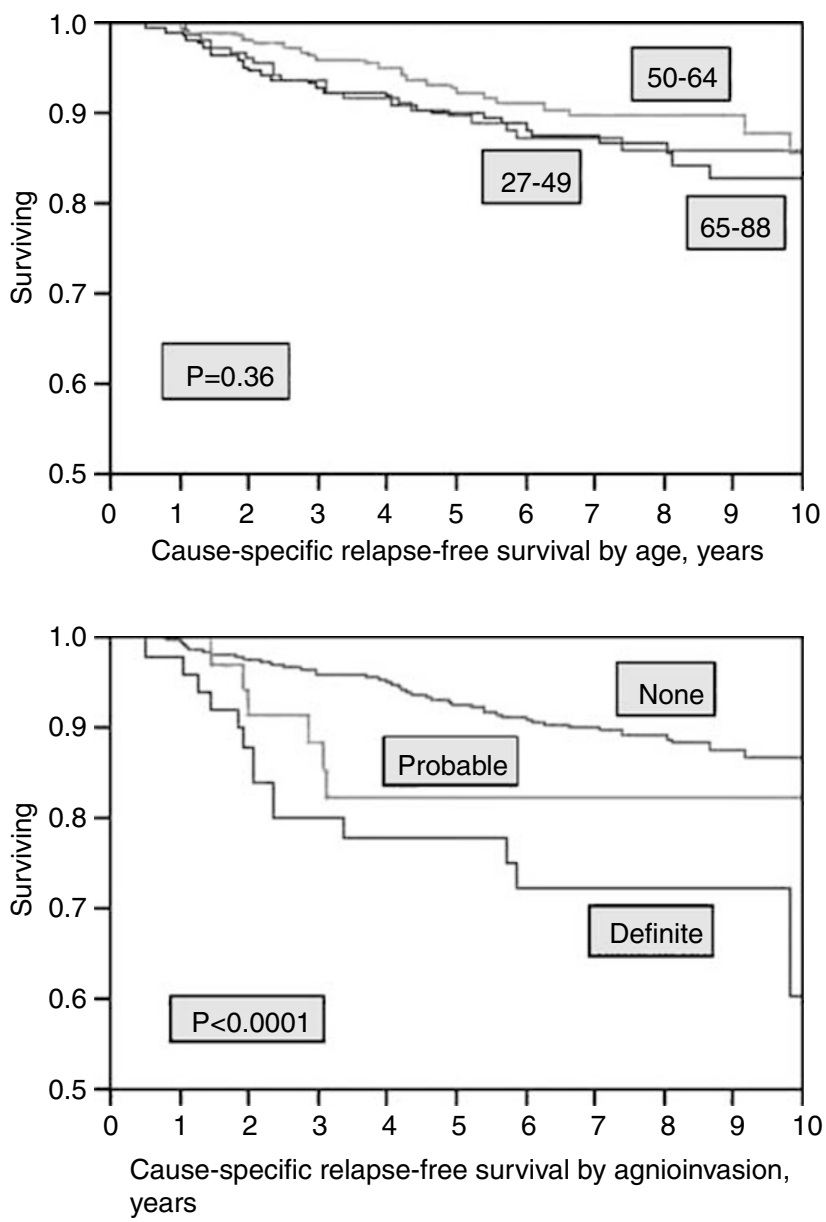

analysis showed a number of univariate predictors for relapse-free tumor-specific survival (Figures 1-3, Table 5). Cutoffs of $0-2,3-10$, and $>10$ mitotic figures per 10 high-power fields (Figure 2) were more effective in determining prognostic groups $(P=0.0093)$ than cutoffs of $0-5,6-10$, and $>10$, which corresponded more closely to those of the Nottingham-Bloom-Richardson system and divided the tumors into groups with a log-rank significance level of $P=0.021$ (survival plot not shown).

Variables with independent predictive power for cause-specific relapse-free survival were tumor size, lymph-angioinvasion, architectural grade, and labeling index (S-phase fraction by thymidine or bromodeoxyuridine uptake) in multivariate analysis (Table 6). Independently predictive variables for cause-specific overall survival were tumor size, lymph-angioinvasion, tubularity, labeling index, and age (Table 7).

When only Nottingham-Bloom-Richardson grading components were entered into the model, of those components only tubularity was independently predictive $(P=0.0095)$. When overall tumor
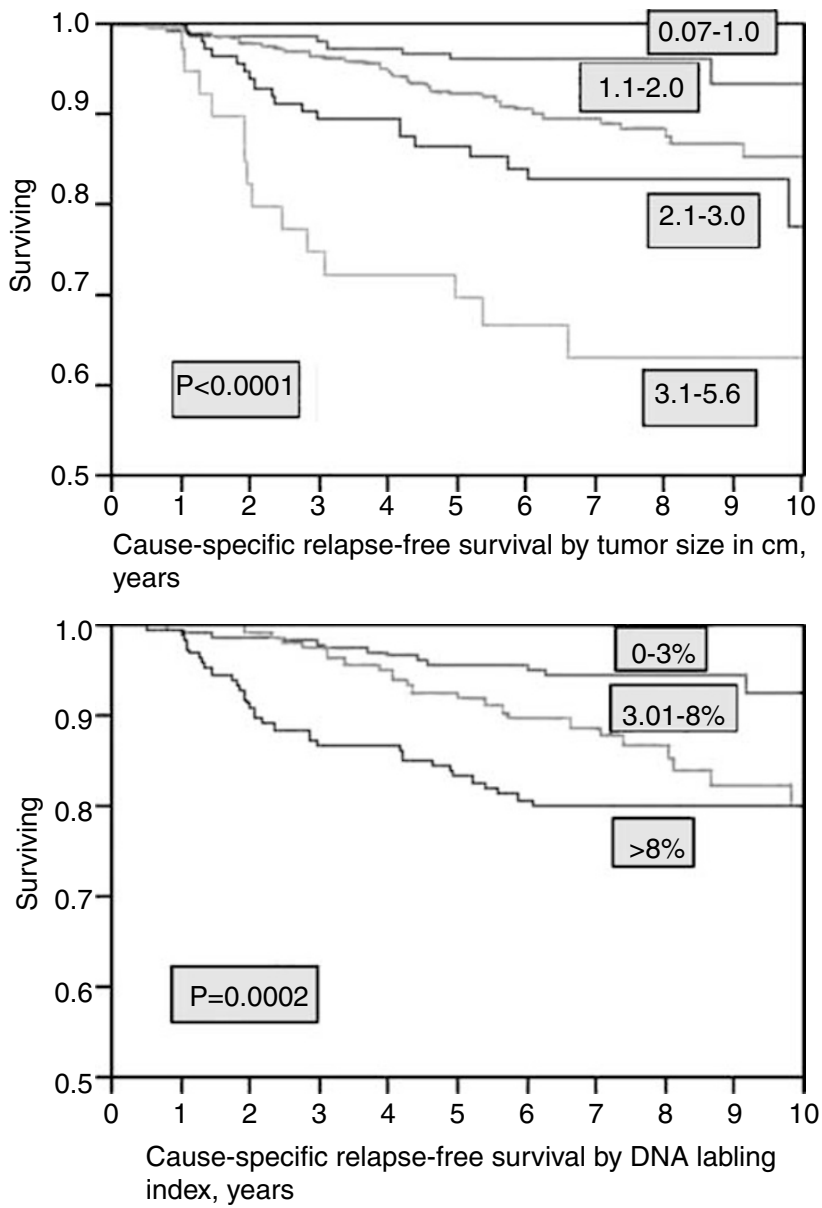

Figure 1 Cause-specific relapse-free survival plot of 631 node-negative breast carcinomas according to age, size of invasive tumor in centimeter, lymphatic or blood vessel invasion, or thymidine/bromodeoxyuridine labeling index. 

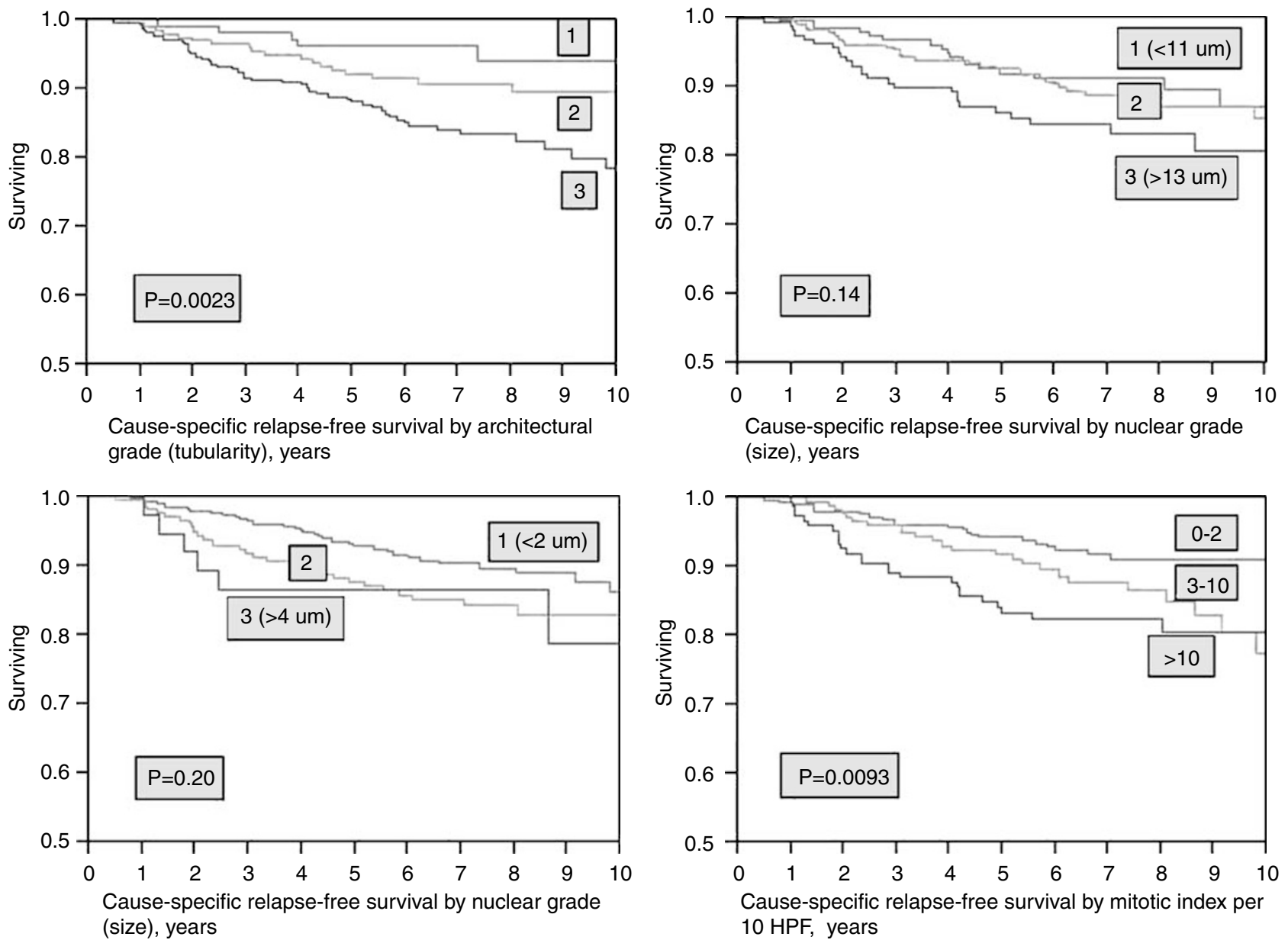

Figure 2 Cause-specific relapse-free survival plot of 631 node-negative breast carcinomas according to tubularity, nuclear size, nucleolar size, and mitotic index.
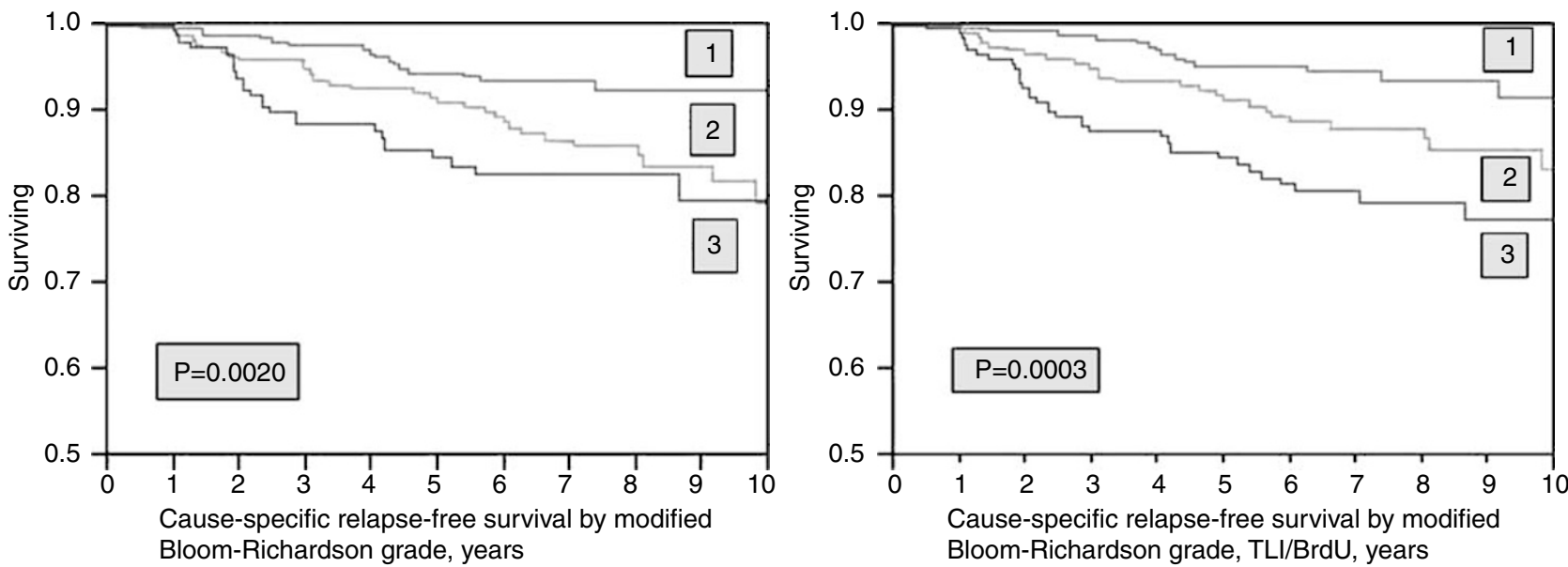

Figure 3 Tumor-specific relapse-free survival plot of 631 node-negative breast carcinomas according to the modified Bloom-Richardson grade and with modified Bloom-Richardson grade mitotic figures per 10 high-power fields replaced by thymidine/bromodeoxyuridine labeling index. Cutoffs for labeling index were 3 and $8 \%$.

grade was entered into the model, it became the only significant predictor $(P<0.05)$, displacing tubularity. When tumor size or labeling index by tritiated thymidine or bromodeoxyuridine uptake were added, either one displaced tumor grade and was the only significant predictor. 
Table 5 Cutoffs for variables in node-negative outcome study

\begin{tabular}{|c|c|c|c|c|c|}
\hline Observation & Cutoffs & $\begin{array}{c}\mathrm{N} \text { of } \\
\text { classes }\end{array}$ & $\begin{array}{c}\mathrm{N} \text { in classes } \\
1 / 2 / 3 / 4^{\mathrm{a}}\end{array}$ & $\begin{array}{c}\text { Events/cens'd } \\
\text { RFS/OS }\end{array}$ & $\begin{array}{c}\text { Log rank prob. } \\
\text { RFS/OS }\end{array}$ \\
\hline Age (years) & 49,65 & 3 & $160 / 235 / 236$ & $75 / 56556 / 575$ & $0.36 / 0.41$ \\
\hline Size tumor $(\mathrm{cm})$ & $1.0,2.0,3.0$ & 4 & $165 / 305 / 120 / 41$ & $75 / 56555 / 574$ & $0.0000 / 0.0000$ \\
\hline Tubularity & $50 \%, 5 \%$ & 3 & $111 / 201 / 319$ & $75 / 56556 / 575$ & $0.002 / 0.002$ \\
\hline Nuclear size $(\mu \mathrm{m})$ & 10,13 & 3 & $184 / 284 / 163$ & $75 / 56556 / 575$ & $0.14 / 0.008$ \\
\hline Nucleolar size $(\mu \mathrm{m})$ & 2,4 & 3 & $378 / 214 / 39$ & $75 / 56556 / 575$ & $0.20 / 0.085$ \\
\hline Mitotic figures per 10 high-power fields & 2,10 & 3 & $291 / 179 / 151$ & $73 / 56548 / 565$ & $0.001 / 0.002$ \\
\hline Tumor necrosis & $0 / 1+, 2+, 3+$ & 3 & $475 / 117 / 39$ & $75 / 56556 / 575$ & $0.016 / 0.014$ \\
\hline Tumor border & $\begin{array}{l}\text { Smth/equiv/ } \\
\text { ragged }^{\mathrm{d}}\end{array}$ & 3 & $88 / 132 / 409$ & $75 / 56554 / 573$ & $0.062 / 0.23$ \\
\hline Bloom-Richardson grade & $1,2,3$ & 3 & 249/221/151 & $73 / 56548 / 565$ & $0.002 / 0.0003$ \\
\hline Bloom-Richardson grade/labeling index ${ }^{\mathrm{e}}$ & $1,2,3$ & 3 & $246 / 200 / 184$ & $75 / 56555 / 574$ & $0.0003 / 0.0001$ \\
\hline Angioinvasion $^{\mathrm{f}}$ & No/eq/yes & 3 & $545 / 36 / 50$ & $75 / 56556 / 575$ & $0.0001 / 0.0001$ \\
\hline Estrogen receptor $(\mathrm{fmol} / \mathrm{mg} \mathrm{p})^{\mathrm{g}}$ & 10,50 & 3 & $144 / 100 / 385$ & $75 / 56554 / 573$ & $0.51 / 0.51$ \\
\hline Progesterone receptor $(\mathrm{fmol} / \mathrm{mg} \mathrm{p})^{\mathrm{g}}$ & 10,50 & 3 & $184 / 107 / 332$ & $74 / 55$ 549/568 & $0.15 / 0.01$ \\
\hline Labeling index ${ }^{\mathrm{h}}$ & $3 \%, 8 \%$ & 3 & $253 / 173 / 204$ & $75 / 56555 / 574$ & $0.0002 / 0.0004$ \\
\hline
\end{tabular}

${ }^{\mathrm{a}}$ Classes as defined by cutoffs.

${ }^{\mathrm{b}}$ Column 1 =events tumor-specific relapse-free survival/overall survival, column 2 =censored cases.

${ }^{\mathrm{C}} P$-values from significance tests on Kaplan-Meier survival plots.

${ }^{\mathrm{d}}$ Border class 1 = smooth, 2 = equivocal, 3 = ragged.

${ }^{\mathrm{e}}$ Bloom-Richardson grade modified by replacement of mitotic index by labeling index with $3,8 \%$ cutoffs.

$\mathrm{f}_{1}=$ no angioinvasion, 2 = equivocal for angioinvasion, $3=$ definite angioinvasion.

$\mathrm{g}_{1}=<10 \mathrm{fmol} /$ specific binding/mg cytosol protein or $<10 \%$ of nuclei staining by immunohistochemistry, $2=10-49 \mathrm{fmol}$ or $10-49 \%$ staining, $3=>49 \mathrm{fmol}$ or $>50 \%$.

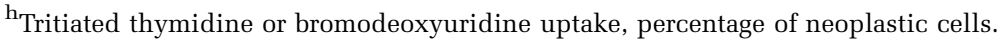

Table 6 Final proportional hazards model for cause-specific relapse-free survival

\begin{tabular}{lccc}
\hline Variable & $\begin{array}{c}\text { Variable } \\
\text { classes }\end{array}$ & $\begin{array}{c}\text { Risk ratio (95\% } \\
\text { confidence limits) }\end{array}$ & Probability $>\chi^{2}$ \\
\hline $\begin{array}{l}\text { Tumor size } \\
\begin{array}{l}\text { Angioinvasion } \\
\text { Architectural }\end{array}\end{array}$ & 3 & $1.76(1.35-2.27)$ & 0.0000 \\
$\begin{array}{l}\text { grade } \\
\text { Labeling } \\
\text { index }\end{array}$ & 3 & $1.56(1.16-2.05)$ & 0.0042 \\
& 3 & $1.34(1.002-1.81)$ & 0.020 \\
\hline
\end{tabular}

Table 7 Final proportional hazards model for cause-specific survival

\begin{tabular}{lccc}
\hline Variable & $\begin{array}{c}\text { Variable } \\
\text { classes }\end{array}$ & $\begin{array}{c}\text { Risk ratio (95\% } \\
\text { confidence limits) }\end{array}$ & Prob $>\chi^{2}$ \\
\hline $\begin{array}{l}\text { Tumor size } \\
\begin{array}{l}\text { Angioinvasion } \\
\text { Architectural }\end{array}\end{array}$ & 4 & $2.04(1.52-2.74)$ & 0.0000 \\
$\begin{array}{l}\text { grade } \\
\text { Labeling }\end{array}$ & 3 & $1.64(1.18-2.21)$ & 0.0044 \\
$\begin{array}{l}\text { index } \\
\text { Age }\end{array}$ & 3 & $1.72(1.13-2.79)$ & 0.011 \\
\hline & 3 & $1.56(1.10-2.25)$ & 0.012 \\
\hline
\end{tabular}

\section{Discussion}

\section{Recognition and Classification of Mitotic Figures}

Agreement was complete on a substantial number of images, showing that participants basically agreed on criteria for recognition of mitotic figures, but reproducibility in classification of mitotic and other nuclear hyperchromatic figures from photographs was in the category of 'fair' by concordance and kappa statistics. Although quality of the photographic prints was judged to be good, lack of concordance may in part be a result of slight loss of resolution in the photographic process and inability to evaluate nuclear figures by throughfocusing. Through-focus digital imaging should be considered for future studies.

Prophases represented a negligible proportion of total mitotic figures recognized. Therefore, miscounting of prophases can account for only a small portion of variability in mitotic counts from one observer to another. Variations in the number of prophases recognized by different observers could be ascribed to differences in opinions as to the degree of chromatin condensation necessary to define a prophase. Occasionally figures thought to be en face metaphase plates by one observer were interpreted by another observer as prophase. Prophase is the longest phase of mitosis, but high resolution is required to recognize $0.2-0.3 \mu \mathrm{m}$ chromosomal filaments present in early prophase. ${ }^{25}$ We recommend that prophases be excluded from mitotic counts performed for cancer grading.

\section{Bloom-Richardson Grading}

\section{Reproducibility}

Our results resemble those of most published studies wherein modest to good levels of agreement 
among independent observers for the NottinghamBloom-Richardson grading system and mostly fair to moderate agreement for its three components have been demonstrated (Table 3). This and other studies show that while disagreements of one step (grade 1 vs grade 2, grade 2 vs grade 3 ) have been common, discrepancies of more than one grade seldom occur. ${ }^{7,26}$ Agreement did not improve in successive trials after review of criteria and group meetings. This outcome suggests that the level of agreement achievable is limited by the subjectivity of grading criteria. Prior studies and ours have shown that agreement is particularly poor for the nuclear component. Nuclear size and nucleolar size and, with more difficulty, nuclear shape are susceptible to quantitative or semiquantitative measurement. No precise standards have been set forth, and measurements are not formally made, in estimating the 'nuclear pleomorphism' of the Bloom-Richardson system. The situation is made complex by inclusion of more than one variable. A nucleus can be small or large, regularly or irregularly contoured, and have a large or small nucleolus. The grader must make a decision, without clear guidance, on how to classify the nucleus with mixed indicators. There is little wonder for poor reproducibility. The mitotic count also has its pitfalls. The common dictum is to count mitotic figures where they are most numerous, but without sampling the whole specimen one does not know where to begin. Nonrandom sampling introduces bias. We recommend selection of areas with maximal cellularity and best nuclear fixation. Finally, mitotic figures can be difficult to distinguish from apoptoses, pyknoses, and irregularities of nuclear staining.

\section{Relative importance of components of \\ Bloom-Richardson grade}

We reviewed 20 studies that compared BloomRichardson grading components and overall grade for prognostic efficacy. Six studies were conducted on node-negative carcinomas, ${ }^{26-31} 13$ on mixed node-negative/positive carcinomas, ${ }^{8,16,32-42}$ and two on node-positive carcinoma. ${ }^{42,43}$ Architecture (tubularity) was the primary independent predictor in three, ${ }^{27,29,43}$ nuclear pleomorphism by subjective assessment in two, ${ }^{33,35}$ morphometric measurements of nuclear area in one, ${ }^{36}$ and mitotic count in eight studies. ${ }^{28,30-32,38-41}$ Mitotic count and overall grade predicted equally in one study. ${ }^{42}$ Mitotic index contributed to prediction along with another grading component in another four studies. ${ }^{8,27,29,36}$ Overall grade was more predictive than any of its components in three studies. ${ }^{8,16,37}$ These published results favor mitotic index as the principal prognostic component of Bloom-Richardson grading systems. Our results also favor mitotic index along with a contribution from tubularity. Furthermore, we found that proliferation index by DNA precursor uptake was superior to mitotic index.
Can the Nottingham-Bloom-Richardson system be improved?

The Nottingham-Bloom-Richardson system placed a disproportionate fraction of our node-negative carcinomas in the low mitotic index category. In 405 tumors for which the exact number of mitotic figures per 10 high-power fields was recorded, $67.7 \%$ were in the low interval, $11.4 \% \mathrm{mid}$, and $20.0 \%$ high. The counts were log-normally distributed with median $=4$, mean $=8.6$, mode $=0$, range 0-113 per 10 high-power fields of $0.18 \mathrm{~mm}^{2}$. Nottingham-Bloom-Richardson system scores 0-6 mitotic figures per 10 high-power fields as 1, 7-12 as 2 , and $>12$ as 3 when corrected for field size of $0.183 \mathrm{~mm}^{2}$. Clayton reported a median of 2.5 mitotic figures/10 high-power fields with field size adjusted to $0.183 \mathrm{~mm} .{ }^{28}$ His mean was 6.5 , and $30 \%$ of tumors had 6.9 or more ${ }^{25}$ Genestie et $a 3^{39}$ reported that only $2 \%$ of the tumors they analyzed, all less than $3 \mathrm{~cm}$ diameter, surpassed the mid to high third Nottingham-Bloom-Richardson cutoff. Medri et $a l^{44}$ recorded $58 \%$ in the low group, $18 \%$ mid, $24 \%$ high. Proliferation index is correlated with tumor size, ${ }^{16}$ but relatively small tumor sizes in these and our studies would not likely account for so uneven a distribution by tertiles when published Nottingham-Bloom-Richardson cutoffs were used. Breast carcinomas with proliferation index in the lower third of the distribution have distinctly better survival results than those in the mid or high thirds. ${ }^{45,46}$ Our cutoffs for labeling index, which divided a large set of invasive breast carcinomas of various stages into equal thirds, ${ }^{47}$ yielded a nearly proportional distribution of $40.2 \%$ low, $27.5 \%$ mid, and $32.4 \%$ high in the node-negative set. Furthermore, we found that a low cutoff of 2 mitotic figures per 10 high-power fields defined a group with very good prognosis (Figure 2) in comparison with the result using standard Nottingham-Bloom-Richardson cutoffs. ${ }^{44}$ Our lower cutoff for labeling index tertiles of $3 \%$ was equivalent to a mitotic index of approximately $3.3 \%$ by correlation analysis of our 404 patients for whom the exact number of mitotic figures per 10 high-power fields was recorded $(r=0.63, P<0.0001)$. For the entire population, a cutoff of 2 mitotic figures per 10 high-power fields with the mid-interval defined by 3-10 mitotic figures per 10 high-power fields gave better separation of relapse-free survival and overall survival plots $(P=0.0093$ and 0.0015 , respectively) than a cutoff of 5, mid-interval 6-10.

More than one multiple of 10 high-power fields must be counted to classify specimens with low mitotic counts reproducibly. For example, given population-frequency one mitotic figure per 10 highpower fields, the binomial theorem predicts a $7 \%$ probability for more than 2 mitotic figures in a single count of 10 high-power fields. If 20 high-power fields are counted and reduced to frequencies per 10 high-power fields with rounding to the lower integer, probabilities would be $1.1 \%$ for a result of 
3 or more. A count of 30 high-power fields would reduce the risk of an error at the critical 2 per 10 high-power fields cutoff to $0.2 \%$. More than 10 highpower fields need not be counted for accurate classification at the 2 per 10 high-power field threshold when the initial result is $>10$ per highpower field.

Theissig et $a l^{47}$ showed superior results when subjective nuclear pleomorphism was replaced by nuclear image cytometry, but this measurement introduces a more complex technology. We reported independent prognostic efficacy of nuclear grading by semiquantitative nuclear measurements through use of an optical grid. ${ }^{48}$ This method bypasses considerations of variation in nuclear size, nuclear features, and chromatin distribution that can complicate reproducibility of 'nuclear pleomorphism' assessment. While nuclear grade is usually the weakest of the Nottingham-Bloom-Richardson components, it seems to be stronger if measured by quantitative methods with potential for reproducibility.

\section{Proliferation Index vs Grade for Prognosis}

\section{DNA precursor uptake}

We used tritiated thymidine and autoradiography for labeling index measurement on the first $20 \%$ of tumors before switching to bromodeoxyuridine immunohistochemistry. The two methods utilize similar biochemical pathways and give equivalent results. ${ }^{21}$ Multivariate analysis demonstrated that the labeling index was an independent predictor of outcome, displacing Bloom-Richardson grade and its components nuclear grade and mitotic index from the multivariate model. Nonetheless, grade was a highly significant univariate predictor. Given that the covariates of outcome were extensively correlated, and interactions with therapy may have existed, ranking of covariates for predictive power might vary from one similar study to another. Our results, together with supporting findings from studies with other proliferation markers (see below), indicate that accurate immunohistochemical measurements of proliferation index will be at least equivalent to tumor grade for prognosis.

Reproducibility of DNA labeling has been demonstrated by replicate counting, ${ }^{49,50}$ replicate sampling, ${ }^{49,51}$ and comparison between primary breast cancer and lymph nodal metastasis. ${ }^{50}$ Approximately $25 \mathrm{~min}$ is required to count 2000 cells visually. A coefficient of variation of $4 \%$ can be achieved by replicate visual counts of 2000 cells when the labeling index is high. Although absolute differences decline with decreasing labeling index, interobserver coefficients of variation increase because of fewer labeled cells counted. ${ }^{49}$ Field selection also contributes to interobserver variability. In all, $61 \%$ of 65 primary breast cancers were regionally heterogeneous for labeling index by criterion of significantly more intersample variance than intrasample variance. ${ }^{51}$ Correlation coefficient for labeling index of breast carcinoma in biopsy $v s$ mastectomy specimen was $r=0.89$, and for primary breast carcinoma vs axillary lymph node metastasis $r=0.90 .{ }^{50}$ Counting larger numbers of cells and from multiple sites within tumors is feasible by semiautomation through image analysis and can be expected to reduce errors, particularly for tumors with low labeling index. Automation is more difficult to adapt to traditional grading. Despite errors introduced into labeling index measurement by visual counting of limited numbers of cells and tumor heterogeneity, labeling index has proven to be sufficiently reliable to demonstrate efficacy in prognosis and prediction of therapeutic response (reviewed by Medri et $a l^{52}$ ).

\section{Choice of method for proliferation index measurement}

The most practical marker for proliferation index is unsettled. Voices for retention of simple mitotic counting can still be heard. ${ }^{31,53}$ We favor further evaluation of immunohistochemical staining procedures because of potential for automation. Uptake of DNA precursors tritiated thymidine or bromodeoxyuridine is attractive because of easy quantitation and excellent biologic rationale. This technique detects cells in the process of DNA synthesis, which is a prerequisite to cell division. It is advantageous over mitotic figure counting because the number of S-phase cells is much larger than that of mitotic cells, and difficulties in distinguishing mitotic cells from apoptoses and other nuclear changes are avoided. Its disadvantage is requirement for incubation of viable tissue, which makes it essentially impractical for routine use. Ki-67 index by MIB-1 antibody measures cycling cells (growth fraction ${ }^{54}$ ), which include $G_{1}, S, G_{2}$ and $M$ cells rather than $S$ phase alone. It is readily quantified, roughly proportional to the bromodeoxyuridine labeling index ${ }^{55}$ has prognostic power at least comparable to that of tumor grade, and resembles DNA precursor uptake in its correlation with histologic and biochemical features of breast carcinoma. ${ }^{56-60}$ The International Breast Cancer Study Group found the MIB-1 Ki-67 index measured by image analysis to be superior to mitotic index. ${ }^{57}$ This appears to be the method of choice as present. The proliferation markers topoisomerase II $\alpha^{45}$ and $\mathrm{KiS}^{46}$ also proved to be better predictors of breast carcinoma outcome than Bloom-Richardson grade. KiS2 is particularly promising because of specificity for the latter portion of the cell cycle, S phase, G2, and M. Cells in $S$ and G2 phases are strongly committed to entering mitosis.

Immunohistochemical measurement of proliferation index is amenable to computer-processed image analysis. Image analysis has been shown to agree nearly perfectly with visual counts for estimation of Ki-67 index. ${ }^{61}$ When evaluated by computerized 
image analysis, the MIB-1 Ki-67 index was an independent prognostic indicator for operable breast carcinoma in general, ${ }^{56}$ and it predicted survival in node-negative patients. ${ }^{57}$ Proliferation index is a marker that now can be measured efficiently. Along with tumor size, axillary lymph nodal status, angiolymphatic invasion, architectural grade (tubular-glandular differentiation), and hormonal receptor and HER2 status it can produce a meaningful phenotypic profile of breast carcinoma. BloomRichardson grade does not add information predictive for outcome to this profile. The profile is readily obtainable in modern pathology laboratories, and with moderate cost.

\section{Other Independent Markers for Node-Negative Breast Carcinoma Prognosis}

We identified tumor size, lymphatic invasion, tubularity and age along with proliferation index as independent predictors of outcome for node-negative breast carcinoma. Tumor grade or mitotic index became independent predictors only when labeling index was omitted from the multivariate model. Tumor size is currently the most powerful single outcome predictor for node-negative cancer. Lymph-angioinvasion is a measure of metastatic potential that becomes more ominous when the tumor is large or the rate of cell proliferation is high. It has related strongly to relapse in node-negative cancer ${ }^{57}$ and notably in small (T1a, T1b) breast carcinomas, ${ }^{29,58}$ in which efficacy of established markers including proliferation index is difficult to demonstrate. Its impact is limited because it is found in only a minority of breast carcinomas; it was detected in $13.4 \%$ of our node-negative tumors. Tubularity also contributed significantly in our patients. The effect of age, which was significant only for overall survival, was minimal.

Other established prognostic markers include proteolytic enzymes, HER2/neu expression, bone marrow assay for epithelial cells, microvascular counts, and gene expression analysis. Estrogen and progesterone receptors and HER2/neu have established roles in prediction of response to therapy. All of these methods can be characterized as phenotypic. Gene transcription assays have shown promising early results. ${ }^{62}$ Which approach, phenotypic or genomic, will combine the best in efficacy and economy is yet to be determined. A recent report describes retrospective use of gene product screening in 668 tamoxifen-treated patients with invasive breast carcinoma and negative axillary lymph nodes to stratify them into three risk categories. ${ }^{63}$ Resultant distant recurrence-free survival plots were substantially similar to relapse-free survival plots of our patients phenotypically and prospectively stratified as low, mid and high DNA labeling index (Figure 1).

\section{Acknowledgements}

Lisa McShane, PhD, Biometric Research Branch, Division of Cancer Treatment and Diagnosis, National Cancer Institute, performed concordance and kappa analyses and offered valuable criticism. Enrique Higa, MD, Julio Lagos, MD, Jose Manes, MD, Joseph Marcus, MD, Roger Reichert, MD, David Scrivner, MD, and Shirley Silbert, MD participated in grading breast carcinomas at St Luke's Hospital.

\section{References}

1 Greenough RB. Varying degrees of malignancy in cancer of the breast. J Cancer Res 1925;9:425-463.

2 Patey DH, Scarff RW. The position of histology in the prognosis of carcinoma of the breast. Lancet 1928;1:801-804.

3 Bloom HJG, Richardson WW. Histological grading and prognosis in breast cancer. Br J Cancer 1957;11: 359-377.

4 Elston CW, Ellis IO. Pathological prognostic factors in breast cancer. I. The value of histological grade in breast cancer: experience from a large study with long-term follow-up. Histopathology 1991;19: 403-410.

5 Elston CW, Ellis IO. The Breast. Churchill-Livingstone: Edinburgh, 1998, pp 367-378.

6 Tavassoli FA, Devilee P. World Health Organization Classification of Tumours: Tumors of the Breast and Female Genital Organs. IARC Press, International Agency for Research on Cancer: Lyon, 2003, pp 18-19.

7 Davis BW, Gelber RD, Goldhirsch A, et al. Prognostic significance of tumor grade in clinical trials of adjuvant therapy for breast cancer with axillary lymph node metastases. Cancer 1986;58:2662-2670.

8 Le Doussal V, Tubiana-Hulin M, Friedman S, et al. Prognostic value of histologic grade nuclear components of Scarff-Bloom-Richardson (SBR). An improved score modification based on a multivariate analysis of 1262 invasive ductal breast carcinomas. Cancer 1989;64:1914-1921.

9 Carriaga MT, Henson DE. The histologic grading of cancer. Cancer 1995;75:406-421.

10 Delides GS, Garas G, Georgouli G, et al. Intralaboratory variations in the grading of breast carcinoma. Arch Pathol Lab Med 1982;106:126-128.

11 Stenkvist B, Bengtsson E, Eriksson O, et al. Histopathological systems of breast cancer classification: reproducibility and clinical significance. J Clin Pathol 1982;36:392-398.

12 Harvey JM, de Klerk NH, Sterrett KGF. Histological grading in breast cancer: interobserver agreement, and relation to other prognostic factors including ploidy. Pathology 1992;24:63-68.

13 Dalton LW, Page DL, Dupont WD. Histologic grading of breast carcinoma. A reproducibility study. Cancer 1994;73:2765-2770.

14 Frierson HF, Wolber RA, Berean KW, et al. Interobserver reproducibility of the Nottingham modification of the Bloom and Richardson histologic grading scheme for infiltrating ductal carcinoma. Am J Clin Pathol 1995;103:195-198. 
15 Robbins P, Pinder S, de Klerk N, et al. Histological grading of breast carcinomas: a study of interobserver agreement. Hum Pathol 1995;26:873-879.

16 Raabe NK, Schistad O, Sauer T, et al. Prognosis of radically operated breast carcinoma patients. A retrospective study of 167 consecutive patients with emphasis on histopathological grading, reproducibility and mean nuclear area. APMIS 1997;105: 363-370.

17 Lakhani SR, Jacquemier J, Sloane J, et al. Multifactorial analysis of differences between sporadic breast cancer and cancers involving BRCA1 and BRCA2 mutations. J Natl Cancer Inst 1998;90:1138-1145.

18 Sikka M, Agarwal S, Ghatia A. Interobserver agreement of the Nottingham histologic grading scheme of nuclear morphometric variables as prognostic predictors in breast cancer. Anticancer Res 1991;11: 1663-1669.

19 Boiesen P, Bendahl PO, Anagnostaki L, et al. Histologic grading in breast cancer-reproducibility between seven pathologic departments. Acta Oncol 2000;39: 41-45.

20 Glass AG, Donis-Keller H, Mies C, et al. The Cooperative Breast Cancer Tissue Resource: archival tissue for the investigation of tumor markers. Clin Cancer Res 2001;7:1843-1849.

21 Meyer JS. Cellular kinetics of breast cancer. In: Donegan WL, Spratt JS (eds). Cancer of the Breast, 5th edn. Saunders: Philadelphia, PA, 2002, pp 377-416.

22 Meyer JS, Province MA. S-phase fraction and nuclear size in long term prognosis of patients with breast cancer. Cancer 1994;74:2287-2299.

23 Fleiss JL. Statistical Methods for Rates and Proportions. John Wiley \& Sons: New York, 1981, pp 212-236.

24 Kaplan EL, Meier P. Nonparametric estimation from incomplete observations. J Am Stat Assoc 1958;53: 457-481.

25 El-Alfy M, Leblond CP. Long duration of mitosis and consequences for the cell cycle concept, as seen in the isthmal cells of the mouse pyloric antrum. I. Identification of early and late steps of mitosis. Cell Tissue Kinet 1987;20:205-213.

26 Page DL, Gray R, Allred DC, et al. Prediction of nodenegative breast cancer outcome by histologic grading and S-phase analysis by flow cytometry. An Eastern Cooperative Oncology Group Study (2192). Am J Clin Oncol 2001;24:10-18.

27 Parl FF, Dupont WD. A retrospective cohort study of histologic risk factors in breast cancer patients. Cancer 1982;50:2410-2416.

28 Clayton F. Pathologic correlates of survival in 378 node-negative infiltrating ductal breast carcinomas. Cancer 1991;68:1309-1317.

29 Lee AKC, Loda M, Mackarem G, et al. Lymph node negative invasive breast carcinoma 1 centimeter or less in size (T1a,bN0M0). Clinicopathologic features and outcome. Cancer 1997;79:761-771.

30 Mandard AM, Denoux Y, Herlin P, et al. Prognostic value of DNA cytometry in 281 premenopausal patients with lymph node negative breast carcinoma randomized in a control trial: multivariate analysis with Ki-67 index, mitotic count, and microvessel density. Cancer 2000;89:1748-1757.

31 Volpi A, Bacci F, Paradiso A, et al. Prognostic relevance of histological grade and its components in node-negative breast cancer patients. Mod Pathol 2004;17:1038-1044.
32 Baak JPA, Van Dop H, Kurver PHJ, et al. The value of morphometry to classic prognosticators in breast cancer. Cancer 1985;56:374-382.

33 Rank F, Dombernowsky P, Jespersen NCB, et al. Histologic malignancy grading of invasive ductal breast carcinoma. A regression analysis of prognostic factors in low-risk carcinomas from a multicenter trial. Cancer 1987;60:1299-1305.

34 Russo J, Frederick J, Ownby HE, et al. Predictors of recurrence and survival of patients with breast cancer. Am J Clin Pathol 1987;88:123-131.

35 Theissig F, Kunze KD, Haroske G, et al. Histological grading of breast cancer. Interobserver reproducibility and prognostic significance. Pathol Res Pract 1990;186:732-736.

36 Uyterlinde AM, Baak JPA, Schipper NW, et al. Further evaluation of the prognostic value of morphometric and flow cytometric parameters in breastcancer patients with long follow-up. Int J Cancer 1990; $45: 1-7$.

37 Aaltomaa S, Lipponen P, Eskelinen ME, et al. The significance of nuclear morphometric variables as prognostic predictors in breast cancer. Anticancer Res 1991;11:1663-1669.

38 Eskelinen M, Lipponen P, Papinaho S, et al. DNA flow cytometry, nuclear morphometry, mitotic indices and steroid receptors as independent prognostic factors in female breast cancer. Int J Cancer 1992;51: 555-561.

39 Genestie C, Zafrani B, Asselain B, et al. Comparison of the prognostic value of Scarff-Bloom-Richardson and Nottingham histological grades in a series of 825 cases of breast cancer: major importance of the mitotic count as a component of both grading systems. Anticancer Res 1998;18:571-576.

40 Thor $\mathrm{AD}$, Liu S, Moore $\mathrm{DH}$, et al. Comparison of mitotic index, in vitro bromodeoxyuridine labeling, and MIB-1 assays to quantitate proliferation in breast cancer. J Clin Oncol 1999;17:470-477.

41 Lynch J, Pattekar R, Barnes DM, et al. Mitotic counts provide additional prognostic information in grade II mammary carcinoma. J Pathol 2002;196:275-279.

42 Clayton F, Hopkins CL. Pathologic correlates of prognosis in lymph-node-positive breast carcinomas. Cancer 1993;71:1780-1790.

43 Maehle BO, Skjaerven R. Prediction of prognosis in lymph node positive breast cancer patients: a statistical study. Br J Surg 1984;71:459-462.

44 Medri L, Volpi A, Nanni O, et al. Prognostic relevance of mitotic activity in patients with node-negative breast cancer. Mod Pathol 2003;16:1067-1075.

45 Rudolph PMG, MacGrogan G, Bonichon F, et al. Prognostic significance of Ki-67 and topoisomerase II $\alpha$ expression in infiltrating ductal carcinoma of the breast. A multivariate analysis of 863 cases. Breast Cancer Res Treat 1999;55:61-71.

46 Rudolph P, Alm P, Heidebrecht H-J, et al. Immunologic proliferation marker Ki-S2 as prognostic indicator for lymph node-negative breast cancer. J Natl Cancer Inst 1999;91:271-278.

47 Theissig F, Dimmer V, Haroske G, et al. Use of nuclear image cytometry, histopathological grading and DNA cytometry to make breast cancer prognosis more objective. Anal Cell Pathol 1991;3:351-360.

48 Meyer JS, Province MA. S-phase fraction and nuclear size in long term prognosis of patients with breast cancer. Cancer 1994;74:2287-2299. 
49 Meyer JS, Bauer WC. Tritiated thymidine labeling index of benign and malignant human breast epithelium. J Surg Oncol 1976;8:165-181.

50 Meyer JS, McDivitt RW. Reliability and stability of the thymidine labeling index of breast carclinoma. Lab Invest 1986;54:160-164.

51 Meyer JS, Wittliff JL. Regional heterogeneity in breast carcinoma: thymidine labelling index, steroid hormone receptors, DNA ploidy. Int J Cancer 1991;47: 213-220.

52 Medri L, Volpi A, Nanni O, et al. Prognostic relevance of mitotic activity in patients with node-negative breast cancer. Mod Pathol 2003;16:1067-1075.

53 Lehr HA, Hansen DA, Kussick S, et al. Assessment of proliferative activity in breast cancer: MIB-1 immunohistochemistry versus mitotic figure count. Hum Pathol 1999;30:1314-1320.

54 Mendelsohn ML. Autoradiographic analysis of cell proliferation in spontaneous breast cancer of $\mathrm{C} 3 \mathrm{H}$ mouse. III. Growth fraction. J Natl Cancer Inst 1962;28: 1015-1030.

55 Goodson III WH, More II DH, Ljung B-M, et al. The functional relationship between in vivo bromodeoxyuridine labeling index and and Ki-67 proliferation index in human breast cancer. Breast Cancer Res Treat 1998;49:155-164.

56 Pinder SE, Wencyk P, Sibbering DM, et al. Assessment of the new proliferation marker MIB1 in breast carcinoma using image analysis: associations with other prognostic factors and survival. Br J Cancer 1995; 71:146-149.

57 Querzoli P, Albonico G, Ferretti S, et al. MIB-1 proliferative activity in invasive breast cancer measured by image analysis. J Clin Pathol 1996;49:868-869.

58 Brown RW, Allred DC, Clark GM, et al. Prognostic value of Ki-67 compared to S-phase fraction in axillary node-negative breast cancer. Clin Cancer Res 1996;2: 585-592.

59 Molino MA, Micciolo R, Turazza M, et al. Ki-67 immunostaining in 322 primary breast cancers: associations with clinical and pathological variables and prognosis. Int J Cancer 1997;74:433-437.

60 Trihia H, Murray S, Price K, et al. Ki-57 expression in breast carcinoma. Its association with grading systems, clinical parameters, and other prognostic factors-A surrogate marker? Cancer 2003;97: 1321-1331.

61 Franklin WA, Bibbo M, Doria MI, et al. Quantitation of estrogen receptor content and Ki-67 staining in breast carcinoma by the microTICAS image analysis system. Anal Quant Cytol Histol 1987;9:279-283.

62 Garber K. Gene expression tests foretell breast cancer's future. Science 2004;303:1754-1755.

63 Paik S, Shak S, Tang F, et al. A multigene assay to predict recurrence of tamoxifen-treated, node-negative breast cancer. N Engl J Med 2004;351:2817-2826. 